\title{
High-sensitivity troponin I and all-cause mortality in patients with stable COPD: an analysis of the COSYCONET study
}

\author{
Benjamin Waschki (10 1,2,3,4 , Peter Alter (103,5, Tanja Zeller ${ }^{1,4}$, \\ Christina Magnussen ${ }^{1,4}$, Johannes T. Neumann ${ }^{1}$, Raphael Twerenbold ${ }^{1}$, \\ Christoph Sinning ${ }^{1}$, Christian Herr ${ }^{6}$, Kathrin Kahnert ${ }^{3,7}$, Sebastian Fähndrich ${ }^{8}$, \\ Stefan Blankenberg ${ }^{1,4}$, Klaus F. Rabe ${ }^{2,3}$, Tobias Welte (103,9, Rudolf A. Jörres ${ }^{3,10}$, \\ Claus F. Vogelmeier ${ }^{3,5}$, Robert Bals (1) $^{6,12}$ and Henrik Watz $z^{3,11,12}$ on behalf of the \\ German COSYCONET Cohort
}

@ERSpublications

High-sensitivity troponin $I$ is a strong predictor of all-cause mortality in patients with stable COPD independent from established mortality predictors of COPD and irrespective of their cardiovascular risk profile http://bit.ly/352ZtDw

Cite this article as: Waschki B, Alter P, Zeller T, et al. High-sensitivity troponin I and all-cause mortality in patients with stable COPD: an analysis of the COSYCONET study. Eur Respir J 2020; 55: 1901314 [https://doi.org/10.1183/13993003.01314-2019].

ABSTRACT Chronic obstructive pulmonary disease (COPD) is a leading cause of death with a considerable part of the population dying from cardiovascular diseases. High-sensitivity troponin I (hsTnI) might help to better identify COPD patients at high risk of mortality. We aimed to study the predictive value of hs-TnI for all-cause mortality beyond established COPD assessments, and after consideration of relevant cardiovascular risk factors and prevalent cardiovascular diseases, in a broad population with stable COPD.

Circulating hs-TnI concentrations together with a wide range of respiratory and cardiovascular markers were evaluated in 2085 patients with stable COPD across all severity stages enrolled in the multicentre COSYCONET cohort study. The primary outcome was all-cause mortality over 3 years of follow-up.

Hs-TnI was detectable in 2020 (96.9\%) patients. The median hs-TnI concentration was $3.8 \mathrm{ng} \cdot \mathrm{L}^{-1}$ (interquartile range $2.5-6.6 \mathrm{ng} \cdot \mathrm{L}^{-1}$ ), with levels above the 99th percentile reference limit of $27 \mathrm{ng} \cdot \mathrm{L}^{-1}$ observed in $1.8 \%$ of patients. In Cox regression analyses including adjustments for airflow limitation, dyspnoea grade, exercise capacity and history of severe exacerbations, as well as traditional cardiovascular risk factors, estimated glomerular filtration rate, ankle-brachial index, $\mathrm{N}$-terminal pro-brain natriuretic peptides and prevalent cardiovascular diseases, hs-TnI was a significant predictor for all-cause mortality, both as a continuous variable (hazard ratio (HR) for $\log$ hs-TnI 1.28, 95\% CI 1.01-1.62) and categorised according to the cut-off of $6 \mathrm{ng} \cdot \mathrm{L}^{-1}$ (HR 1.63, 95\% CI 1.10-2.42).

In patients with stable COPD, hs-TnI is a strong predictor of all-cause mortality beyond established COPD mortality predictors, and independent of a broad range of cardiovascular risk factors and prevalent cardiovascular diseases. Hs-TnI concentrations well below the upper reference limit provide further prognostic value for all patients with COPD when added to established risk assessments.

This article has supplementary material available from erj.ersjournals.com

The study is registered at clinicaltrials.gov with identifier number NCT01245933.

Received: 03 July 2019 | Accepted after revision: 11 Nov 2019

Copyright OERS 2020 


\section{Introduction}

Chronic obstructive pulmonary disease (COPD) is a usually progressive disease of the airways, the alveoli and the microvasculature, which is frequently associated with cardiovascular diseases [1]. Currently, COPD is responsible for $>3$ million deaths per year worldwide, making it the third leading cause of death, just behind ischaemic heart disease and stroke [2]. The identification of patients at high mortality risk and a better pathophysiological understanding of underlying phenotypes is essential to facilitate early intervention with appropriate treatment to reduce mortality in COPD $[2,3]$.

Patients with COPD have a 2.5 -fold increased risk of cardiovascular disease [4]. Furthermore, up to one-third of patients with COPD die from cardiovascular disease, making this a leading cause of death in patients with mild and moderate COPD $[5,6]$. Indeed, cardiovascular markers such as the ankle-brachial index or computed tomography coronary artery calcium scoring have been shown to predict all-cause mortality in patients with COPD $[7,8]$. However, these markers are cumbersome, and early use of simple and objective cardiovascular assessments may help to improve risk stratification in COPD [2].

Cardiac troponins, i.e. the isoforms of troponin I and troponin $\mathrm{T}$, are released in the heart as a result of myocardial injury or necrosis and are therefore established markers for the diagnosis of acute myocardial infarction [9-12]. Whereas the expression of troponin $\mathrm{T}$ has been found occasionally in diseased skeletal muscle, troponin I is expressed exclusively in the myocardium [11]. Slight increases in troponin levels could be detected in chronic diseases such as coronary artery disease, heart failure and chronic kidney disease, and improve risk stratification [13-15]. Modern high-sensitivity troponin assays allow the detection of very low circulating troponin concentrations even in general population [16]. Moreover, high-sensitivity troponin levels predict mortality beyond existing cardiovascular risk factors in population based studies, even with cut-offs well below that used for the diagnosis of myocardial infarction [17]. In patients with stable COPD, only two studies analysing cardiac troponins and longitudinal outcomes have been completed so far, both conducted in selected populations $[18,19]$. To our knowledge, the association between high-sensitivity troponin I (hs-TnI) and all-cause mortality has not been studied before within a broad COPD cohort that includes all COPD severities and does not preselect for cardiovascular disease.

We hypothesised that hs-TnI might be useful beyond existing risk factors to identify patients with stable COPD who are at high risk of mortality. Therefore, our primary aim was to study the predictive value of hs-TnI for all-cause mortality beyond established COPD mortality predictors and after correction for established cardiovascular risk factors and prevalent cardiovascular diseases. A secondary aim was to identify independent determinants of higher hs-TnI levels.

\section{Methods \\ Study design and patients}

This report describes a longitudinal observation analysis that involved 2085 patients with stable COPD from the ongoing COSYCONET (COPD and Systemic Consequences-Comorbidities Network) cohort study. The overall aims and methods of COSYCONET have been described previously [20]. In brief, a central purpose of COSYCONET is to analyse the impact of extrapulmonary disorders on morbidity and mortality of patients with COPD [20]. Patients were recruited in 31 German study centres primarily following referral from respiratory specialists and primary-care practitioners. Inclusion criteria were broad with minimal exclusion criteria applied in order to cover a wide range of patterns of the disease [20]. A total of 2741 stable patients were included and underwent a broad panel of assessments with priority given to the assessment of pulmonary function and cardiovascular comorbidities. For the present report, we

Affiliations: ${ }^{1}$ Dept of General and Interventional Cardiology, University Heart Center Hamburg, Hamburg, Germany. ${ }^{2}$ LungenClinic Grosshansdorf, Airway Research Center North (ARCN), Grosshansdorf, Germany. ${ }^{3}$ German Center for Lung Research (DZL). ${ }^{4}$ German Center for Cardiovascular Research (DZHK), partner site Hamburg/Kiel/Lübeck. ${ }^{5}$ Dept of Medicine, Pulmonary and Critical Care Medicine, Philipps-University of Marburg (UMR), Marburg, Germany. ${ }^{6}$ Dept of Internal Medicine V - Pulmonology, Allergology, Critical Care Care Medicine, Saarland University Hospital, Homburg, Germany. ${ }^{7}$ Dept of Internal Medicine V. University of Munich (LMU), Comprehensive Pneumology Center, Munich, Germany. ${ }^{8}$ Dept of Pneumology, University Medical Center Freiburg, Freiburg, Germany. ${ }^{9}$ Dept of Respiratory Medicine, Hannover Medical School, Research in Endstage and Obstructive Lung Disease Hannover (BREATH), Hannover, Germany. ${ }^{10}$ Institute and Outpatient Clinic for Occupational, Social and Environmental Medicine, University Hospital, Ludwig Maximilians University (LMU) Munich, Comprehensive Pneumology Center Munich (CPC-M), Munich, Germany. ${ }^{11}$ Pulmonary Research Institute at LungenClinic Grosshansdorf, ARCN, Grosshansdorf, Germany.

${ }^{12}$ Both authors contributed equally.

Correspondence: Benjamin Waschki, Dept of General and Interventional Cardiology, University Heart Center Hamburg, Germany and LungenClinic Grosshansdorf, Airway Research Center North (ARCN), German Center for Lung Research (DZL), Grosshansdorf, Germany. E-mail: b.waschkiduke.de 
included patients with a confirmed Global Initiative for Chronic Obstructive Lung Disease (GOLD) stages I-IV. Additional details and a patient flow chart are given in supplementary figure E1. COSYCONET was approved by the ethical committees of all study centres and all patients gave written informed consent.

\section{Troponin and other laboratory measurements}

The laboratory measurements described in this analysis were obtained from blood samples collected at baseline. Routine laboratory parameters were determined at each local laboratory. N-terminal pro-brain natriuretic peptide (NT-proBNP) was measured from stored blood samples using a multiplex assay (MILLIPLEX, Merck Millipore, Darmstadt, Germany) in the COSYCONET biobank at the Saarland University Hospital (Homburg, Germany). Troponin I was measured using a high-sensitivity assay (Architect STAT, Abbott Diagnostics, Wiesbaden, Germany) at the University Heart Center (Hamburg, Germany). The assay range is $0-50000 \mathrm{ng} \cdot \mathrm{L}^{-1}$ and a validated lower limit of detection is $1.2 \mathrm{ng} \cdot \mathrm{L}^{-1}$ [19]. Observed values below this limit were included for subsequent analyses. The concentration representing the 99th percentile derived in a reference population was $27 \mathrm{ng} \cdot \mathrm{L}^{-1}$; this represents the upper reference limit and is used for the diagnosis of myocardial infarction [21]. A much lower hs-TnI cut-off of $6 \mathrm{ng} \cdot \mathrm{L}^{-1}$ has been shown to identify individuals in the general population who are at high risk of death during follow-up independent from established risk factors [17]. We therefore used the cut-off of $6 \mathrm{ng} \cdot \mathrm{L}^{-1}$ to dichotomise high- versus low-risk patients in the present analysis.

\section{COPD assessments}

We assessed airway obstruction (post-bronchodilator forced expiratory volume in $1 \mathrm{~s}\left(\mathrm{FEV}_{1}\right)$, dyspnoea (modified Medical Research Council (mMRC) scale), exercise capacity (6-min walk distance (6MWD)), history of exacerbations, hypoxaemia and hypercapnia, as stated in the supplementary material and as described previously [20]. The BODE index (body mass index (BMI), airway obstruction, dyspnoea and exercise capacity), which is the most frequently used prognostic assessment in COPD, was calculated, with a cut-off of $>4$ used to dichotomise the index [22].

\section{Assessment of cardiovascular risk factors and cardiovascular diseases}

History of myocardial infarction, history of stroke and presence of coronary artery disease were systematically recorded in a structured interview [20]. A combination of self-reported comorbidities and objective measurements was the basis for the definition of hypertension, diabetes and hyperlipidaemia [23, 24]. All other cardiovascular risk factors and diseases were defined solely on the basis of objective measurements, i.e. estimated glomerular filtration rate (eGFR), ankle-brachial index, high-sensitivity C-reactive protein (hs-CRP), NT-proBNP, left ventricular ejection fraction and tricuspid annular plane systolic excursion [24-30]. Additional details are provided in the supplementary material.

\section{Outcome}

The primary outcome of this report was all-cause mortality over 3 years of follow-up. After baseline, patients were invited for each follow-up visit via telephone and letters. If a patient missed a follow-up visit without formally withdrawing from the study, research assistants ascertained the survival status by contacting partners, relatives, primary care practitioners and hospitals. Additional details are provided in the supplementary material.

\section{Statistical analyses}

For the descriptive analyses hs-TnI was categorised into quartiles. For the further analyses hs-TnI was both dichotomised according to the cut-off of $6 \mathrm{ng} \cdot \mathrm{L}^{-1}$, and was used as a continuous variable (after log-transformation). Linear trends in patient demographics, cardiovascular markers and COPD assessments across the hs-TnI quartiles were analysed using Chi-squared tests for dichotomised variables and ANOVA or Kruskal-Wallis test for continuous variables. In addition, the association between continuous hs-TnI values and COPD assessments was tested after adjustment for the traditional cardiovascular risk factors age, sex, smoking status, hypertension and hyperlipidaemia along with the presence of coronary artery disease and history of myocardial infarction using separate logistic regression analyses [24].

To identify the best model of independent predictors for hs-TnI levels $>6 \mathrm{ng} \cdot \mathrm{L}^{-1}$ among cardiovascular markers and COPD assessments, we performed a logistic regression analysis with backward elimination including all variables which showed $\mathrm{p}<0.10$ on the univariate level.

The relationship between hs-TnI quartiles and all-cause mortality was analysed by Kaplan-Meier survival plots and log-rank tests. To calculate the relative risk of mortality associated with hs-TnI levels, we used a series of four Cox proportional hazard regression models, adjusting stepwise for established mortality predictors of COPD, cardiovascular risk factors and prevalent cardiovascular diseases. The first model was 
unadjusted. The second was adjusted for established mortality predictors in COPD, specifically the components of the BODE score (BMI, $\mathrm{FEV}_{1}, \mathrm{mMRC}$ and $\left.6 \mathrm{MWD}\right)$ plus age, hypoxaemia, hypercapnia, presence of severe exacerbations in the prior year and a correction for $\alpha_{1}$-antitrypsin deficiency. The third model added further adjustments for the cardiovascular risk factors sex, hypertension, diabetes, smoking status, hyperlipidaemia, eGFR and log hs-CRP. The final model added adjustments for cardiovascular diseases, i.e. coronary artery disease, history of myocardial infarction, history of stroke, decreased anklebrachial index and log NT-proBNP. All Cox analyses were performed twice, first including continuous log-transformed hs-TnI values and second with hs-TnI dichotomised with the cut-off of $6 \mathrm{ng} \cdot \mathrm{L}^{-1}$.

Finally, we calculated composite variables from established mortality predictors in COPD (i.e. $\mathrm{FEV}_{1}$ and BODE index) and hs-TnI to visualise the effect of hs-TnI as an additional predictor of survival, and in particular the relative risk in patients with high hs-TnI and high BODE index compared to those with low hs-TnI and high BODE index. The added discriminative power offered by the addition of hs-TnI to $\mathrm{FEV}_{1}$ and to the BODE score was analysed using $\mathrm{C}$ statistics [31]. Additional details are provided in the supplementary material.

\section{Results}

Hs-Tnl levels in COPD

Among 2085 patients with COPD available for hs-TnI analyses, 2020 (96.9\%) had hs-TnI concentrations above the limit of detection. Of those, $38(1.8 \%)$ patients had levels that exceeded the upper reference limit of $27 \mathrm{ng} \cdot \mathrm{L}^{-1}$. The median hs-TnI level was $3.8 \mathrm{ng} \cdot \mathrm{L}^{-1}$ (interquartile range (IQR) 2.5-6.6 ng. $\mathrm{L}^{-1}$ ).

\section{Associations with cardiovascular risk factors, prevalent cardiovascular diseases and COPD assessments}

There were significant differences in patients' demographics, cardiovascular risk factors, cardiovascular diseases and concomitant cardiovascular medication across hs-TnI quartiles (table 1). In addition, we observed differences in COPD assessments across hs-TnI quartiles, with significant associations for $\mathrm{FEV}_{1}$, mMRC, severe exacerbations in the prior year (but not mild or moderate exacerbations), 6MWD, BODE index, hypoxaemia and hypercapnia on the univariate level (table 1). After adjustment for prevalent coronary artery disease, history of myocardial infarction and the traditional cardiovascular risk factors age, sex, smoking, hypertension and hyperlipidaemia, hs-TnI remained significantly associated with airflow limitation, mMRC score, 6MWD, BODE score, history of one or more severe exacerbations, hypoxaemia and hypercapnia (table 2). Further results of bivariate associations are given in the supplementary material.

\section{Independent determinants of higher hs-Tnl levels in COPD}

We found hs-TnI levels above the cut-off of $6 \mathrm{ng} \cdot \mathrm{L}^{-1}$ in $595(29 \%)$ patients. Among all cardiovascular markers and COPD assessments with a relevant univariate association with hs-TnI quartiles, the best independent predictors for hs-TnI concentrations $>6 \mathrm{ng} \cdot \mathrm{L}^{-1}$ were higher age, male sex, the presence of hypertension, history of angina pectoris, NT-proBNP level, $\mathrm{mMRC} \geqslant 2$, one or more severe exacerbations in the prior year and presence of right ventricular dysfunction (table 3 ).

\section{Predictive value of hs-Tnl for all-cause mortality in COPD}

Out of 2045 COPD patients with baseline hs-TnI measurement who also were available for a follow-up, 136 (6.7\%) patients died (median follow-up period 36 months, IQR 21-37 months). Figure 1 shows survival curves across quartiles of hs-TnI levels. There was a strong association between hs-TnI quartiles and all-cause mortality $(\mathrm{p}<0.001)$. Patients in the highest quartile had a significantly higher mortality risk compared to both those in the lowest quartile (hazard ratio (HR) 2.42, 95\% CI 1.45-4.04; p<0.001) and those in the second lowest quartile (HR 1.76, 95\% CI 1.10-2.80; $\mathrm{p}=0.018$ ). The mortality risk of patients in the lowest quartile differed significantly from those in the highest quartile and those in the second highest quartile.

Table 4 depicts the mortality risk associated with hs-TnI from a series of Cox regression analyses, adjusting stepwise for established mortality predictors of COPD, cardiovascular risk factors and prevalent cardiovascular diseases. After adjusting for the established COPD assessments, hs-TnI added independent information regarding all-cause mortality (table 4, model 2). After further adjustments for the cardiovascular risk factors, hs-TnI remained a significant predictor of all-cause mortality (table 4, model 3). In the final model, including all previous adjustments plus further adjustments for prevalent cardiovascular diseases, the independent association between hs-TnI and all-cause mortality was further attenuated, but remained significant for hs-TnI as both a continuous variable and categorised according to the cut-off of $6 \mathrm{ng} \cdot \mathrm{L}^{-1}$ (table 4, model 4). In contrast to hs-TnI, the inclusion of NT-proBNP did not significantly improve risk prediction in the final model (table 4, model 4).

Figure 2 displays combined variables to visualise the predictive value of high hs-TnI beyond that of the established mortality predictors in COPD, i.e. $\mathrm{FEV}_{1}$ (figure $2 \mathrm{a}$ ) and BODE score (figure $2 \mathrm{~b}$ ). Patients with 
TABLE 1 Patients' demographics, cardiovascular risk factors, cardiovascular diseases and chronic obstructive pulmonary disease (COPD) assessments according to quartiles of high-sensitivity troponin I

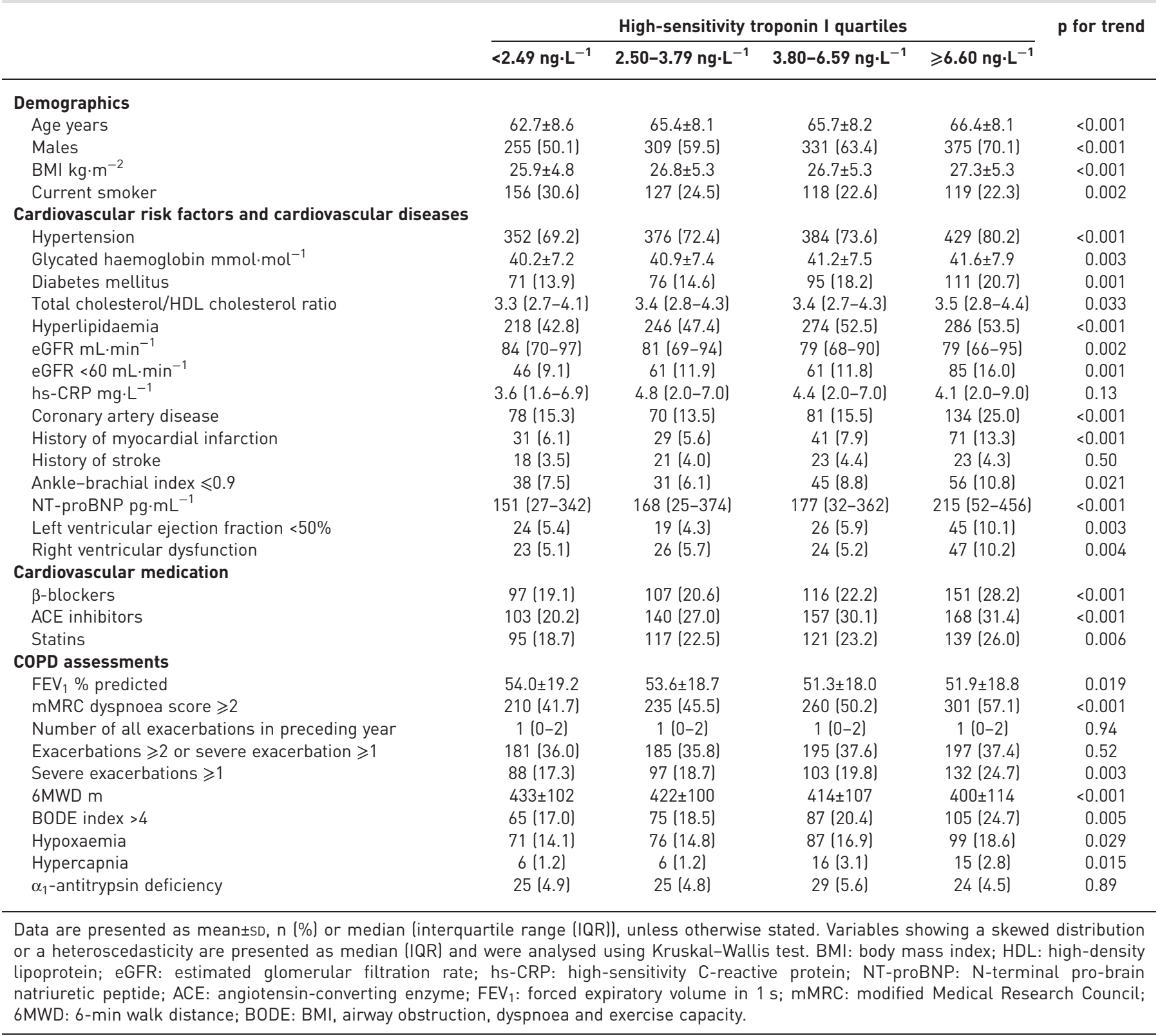

high BODE index and hs-TnI $>6 \mathrm{ng} \cdot \mathrm{L}^{-1}$ had a 2.6-fold higher risk of all-cause mortality compared to those patients with high BODE index but low hs-TnI (HR 2.56, 95\% CI 1.39-4.74; $\mathrm{p}=0.003$ ) (figure 2b). The predictive value determined by the $\mathrm{C}$ statistic also improved significantly when hs-TnI was added to $\mathrm{FEV}_{1}$ in comparison to $\mathrm{FEV}_{1}$ alone (C statistic 0.682 versus $0.661, \mathrm{p}$-value for the change 0.012 ) and when hs-TnI was added to the BODE score in comparison to the BODE score alone (C statistic 0.691 versus 0.670 , p-value for the change $<0.001$ ).

Last, we analysed the predictive value of hs-TnI for future exacerbations of COPD. There was no significant association between log-transformed hs-TnI and future exacerbations (OR 0.94, 95\% CI 0.841.06; $\mathrm{p}=0.31$ ) or exacerbation-related hospitalisations (OR 1.05, 95\% CI 0.92-1.19; $\mathrm{p}=0.51$ ).

\section{Discussion}

The main finding of our study is that hs-TnI is a strong predictor of all-cause mortality in patients with stable COPD beyond established COPD mortality predictors, and after correction for a wide range of 
TABLE 2 Adjusted associations between high-sensitivity troponin I (hs-Tnl) and chronic obstructive pulmonary disease (COPD) assessments

\begin{tabular}{|c|c|c|c|c|c|c|c|}
\hline & $\begin{array}{c}\text { Airflow } \\
\text { limitation } \\
\text { [GOLD stage } \\
\text { III or IV] }\end{array}$ & $\begin{array}{l}\text { Dyspnoea } \\
\text { grade } \\
\text { (mMRC } \\
\text { score } \geqslant 2 \text { ) }\end{array}$ & $\begin{array}{l}\text { Exercise } \\
\text { capacity } \\
\text { (6MWD } \\
<350 \mathrm{~m} \text { ) }\end{array}$ & $\begin{array}{c}\text { BODE index } \\
\text { (BODE score } \\
>4 \text { ] }\end{array}$ & $\begin{array}{l}\text { Exacerbations } \\
\quad l \geqslant 1 \text { severe } \\
\text { exacerbation })\end{array}$ & $\begin{array}{c}\text { Hypoxaemia } \\
\left(P_{\mathrm{aO}_{2}}\right. \\
<55 \mathrm{mmHg})\end{array}$ & $\begin{array}{c}\text { Hypercapnia } \\
\left(P_{\mathrm{aco}_{2}}\right. \\
\geqslant 50 \mathrm{mmHg})\end{array}$ \\
\hline $\begin{array}{l}\text { Adjusted } \mathrm{OR}^{\#}(95 \% \mathrm{Cl}) \\
\text { for log hs-TnI ng. } \mathrm{L}^{-1}\end{array}$ & $\begin{array}{c}1.15 \\
(1.03-1.29)\end{array}$ & $\begin{array}{c}1.29 \\
(1.14-1.45)\end{array}$ & $\begin{array}{c}1.20 \\
(1.05-1.38)\end{array}$ & $\begin{array}{c}1.19 \\
(1.01-1.39)\end{array}$ & $\begin{array}{c}1.20 \\
(1.05-1.38)\end{array}$ & $\begin{array}{c}1.19 \\
(1.03-1.38)\end{array}$ & $\begin{array}{c}1.56 \\
(1.10-2.20)\end{array}$ \\
\hline p-value & 0.016 & $<0.001$ & 0.010 & 0.040 & 0.007 & 0.022 & 0.012 \\
\hline
\end{tabular}

GOLD: Global Initiative for Chronic Obstructive Lung Disease; mMRC: modified Medical Research Council; 6MWD: 6-min walk distance; BODE: body mass index, airway obstruction, dyspnoea and exercise capacity; $P_{\mathrm{aO}_{2}}$ : arterial oxygen tension; $P_{\mathrm{aCO}_{2}}$ : arterial carbon dioxide tension. ${ }^{\#}$ : odds ratios and corresponding $\mathrm{p}$-values for log-transformed hs-Tnl as predictor for different COPD assessments calculated by separate logistic regression analyses are given. Each column represents a separate analysis with the dependent variable stated in brackets. All analyses were adjusted for age, sex, smoking status, hypertension, hyperlipidaemia, coronary artery disease and history of myocardial infarction.

cardiovascular risk factors and prevalent cardiovascular diseases. In addition, we found hs-TnI to be cross-sectionally associated with key COPD assessments, and identified a combination of cardiovascular and respiratory markers that were the best independent predictors of higher levels of hs-TnI in our COPD cohort. Therefore, hs-TnI may improve risk stratification in all patients with stable COPD when added to established risk predictors.

Previously, circulating hs-TnI has been studied in a distinct COPD population with moderate airflow limitation and prevalent cardiovascular disease or increased cardiovascular risk [19]. Here we showed for the first time that circulating hs-TnI is detectable in the vast majority (i.e. 97\%) of patients with stable COPD across all severity stages and irrespective of their cardiovascular risk profile. The proportion of patients in our cohort with hs-TnI concentrations above the 99th percentile upper reference limit of $27 \mathrm{ng} \cdot \mathrm{L}^{-1}$ (i.e. $1.8 \%$ ) was slightly higher than in the general population (i.e. $1.0 \%$, by definition). The proportion of our patients with hs-TnI concentrations above the cut-off of $6 \mathrm{ng} \cdot \mathrm{L}^{-1}$ was clearly higher than in the general population (29\% versus $~ 15 \%)$ [17]. This is consistent with the finding that patients with stable COPD have higher troponin T levels compared to matched controls [32].

In addition, our data show the relationship of hs-TnI with a broad range of cardiovascular risk factors and prevalent cardiovascular diseases in COPD. However, we found hs-TnI to be associated with airflow limitation, dyspnoea, exercise capacity, BODE index, history of severe exacerbations, hypoxaemia and hypercapnia, independent of traditional cardiovascular risk factors and prevalent coronary artery disease. An independent association between airflow limitation and hs-TnI has been reported by ADAMSON et al. [19]. Similarly, associations between hs-troponin $\mathrm{T}$ and some of the other COPD assessments have previously been shown in smaller studies in patients with stable COPD [32, 33]. However, in contrast to our report, ADAmSON et al. [19] did not find hs-TnI to be associated with prior exacerbations in their

TABLE 3 Independent determinants of high-sensitivity troponin I (hs-Tnl) levels $>6 \mathrm{ng} \cdot \mathrm{L}^{-1}$ in patients with stable chronic obstructive pulmonary disease

\begin{tabular}{lcc} 
& OR (95\% CI) & p-value \\
\hline Age & $1.02(1.00-1.03)$ & 0.014 \\
Female sex & $0.60(0.48-0.76)$ & $<0.001$ \\
Hypertension & $1.49(1.14-1.94)$ & 0.003 \\
History of myocardial infarction & $1.50(1.05-2.15)$ & 0.028 \\
NT-proBNP log transformed pg·mL & $1.10(1.03-1.18)$ & 0.004 \\
Right ventricular dysfunction & $1.58(1.06-2.36)$ & 0.025 \\
mMRC dyspnoea score $\geqslant 2$ & $1.44(1.16-1.80)$ & 0.001 \\
One or more severe exacerbations in preceding year & $1.41(1.08-1.83)$ & 0.010 \\
\hline
\end{tabular}

The model represents the final multivariate logistic regression analysis with $\mathrm{hs}-\mathrm{Tnl}>6 \mathrm{ng} \cdot \mathrm{L}^{-1}$ as the dependent variable. The independent predictors stated here remained within a multivariate logistic regression analysis with a backward elimination starting with all variables showing at least a trend for significant differences across hs-Tnl quartiles stated in table 1. NT-proBNP: N-terminal pro-brain natriuretic peptide; mMRC: modified Medical Research Council. 
FIGURE 1 Kaplan-Meier survival curves of patients with stable chronic obstructive pulmonary disease according to quartiles of high-sensitivity troponin I (hs-Tnl). Log-rank test $p<0.001$.

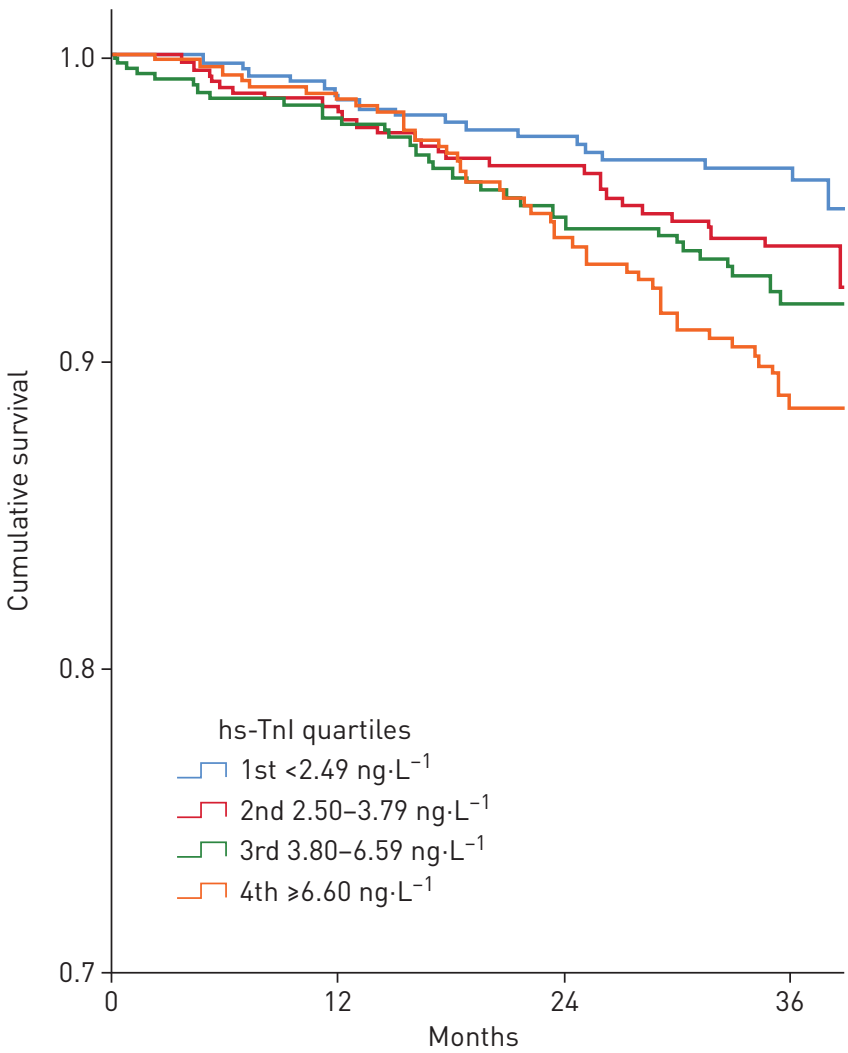

subgroup study. The most likely reasons for this discrepancy are an overall low frequency of prior exacerbations in that study and that the authors combined moderate and severe exacerbations as one end-point [19]. Indeed, we found a history of severe exacerbations, but not milder exacerbations, to be significantly associated with hs-TnI.

Among the COPD assessments, both MMRC score and severe exacerbations in the prior year remained independent predictors for higher hs-TnI levels along with a history of myocardial infarction, higher NT-proBNP levels, right ventricular dysfunction, and the established cardiovascular risk factors age, sex

TABLE 4 Association between high-sensitivity troponin I (hs-Tnl) and all-cause mortality in stable chronic obstructive pulmonary disease (COPD)

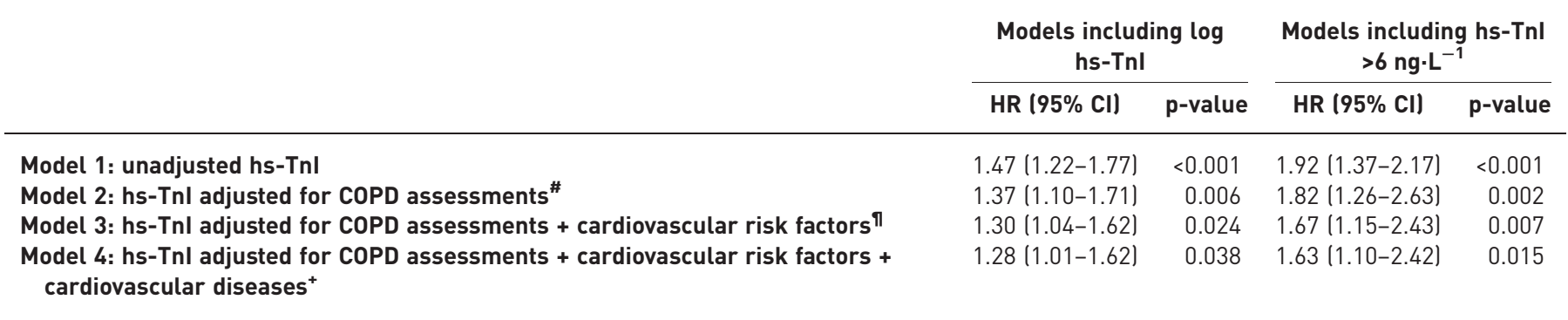

Eight different Cox regression analyses are given, four including log-transformed hs-Tnl as continuous variable and four including dichotomised hs-Tnl with the cut-off $6 \mathrm{ng} \cdot \mathrm{L}^{-1}$ as independent predictor. Hazard ratios (HR) for hs-Tnl as continuous variable are given for each unit increase in the natural logarithm. ${ }^{\#}$ : log hs-Tnl or hs-Tnl $>6 \mathrm{ng} \cdot \mathrm{L}^{-1}$ adjusted for age, body mass index, forced expiratory volume in $1 \mathrm{~s}$, modified Medical Research Council scale, 6-min walk distance, one or more severe exacerbations in the prior year, hypoxaemia, hypercapnia and $\alpha_{1}$-antitrypsin deficiency (121 observed deaths out of 1938 patients); ${ }^{\Uparrow}:$ log hs-Tnl or hs-Tnl $>6 \mathrm{ng} \cdot \mathrm{L}^{-1}$ adjusted for all variables included in model 2 plus sex, hypertension, diabetes, smoking status, hyperlipidaemia, estimated glomerular filtration rate and log high-sensitivity C-reactive protein (121 observed deaths out of 1917 patients); ${ }^{+}:$log hs-Tnl or hs-Tnl $>6 \mathrm{ng} \cdot \mathrm{L}^{-1}$ adjusted for all variables included in model 3 plus coronary artery disease, history of myocardial infarction, history of stroke, ankle-brachial index $\leqslant 0.90$ and log $\mathrm{N}$-terminal pro-brain natriuretic peptide (NT-proBNP) (117 observed deaths out of 1870 patients). The corresponding hazard ratios for log NT-proBNP within model 4 were $1.07(0.95-1.20), p=0.29$ and $1.06(0.94-1.20), p=0.33$, respectively. 
a)

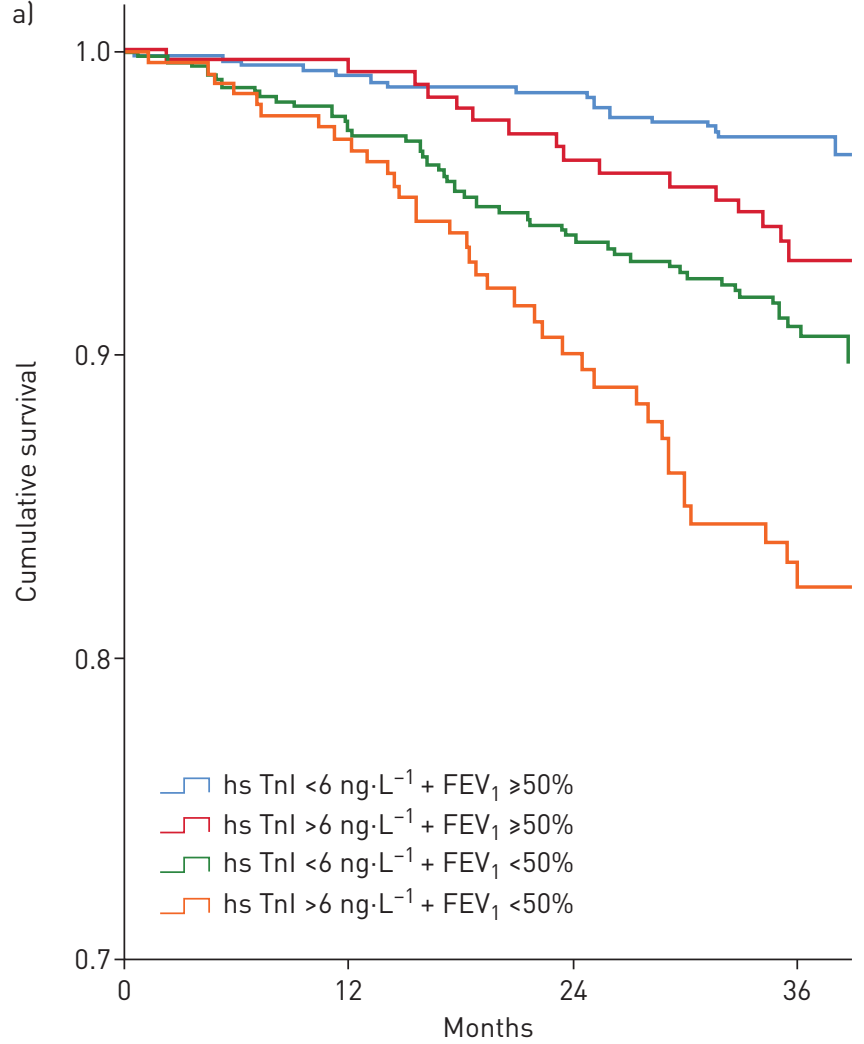

b)

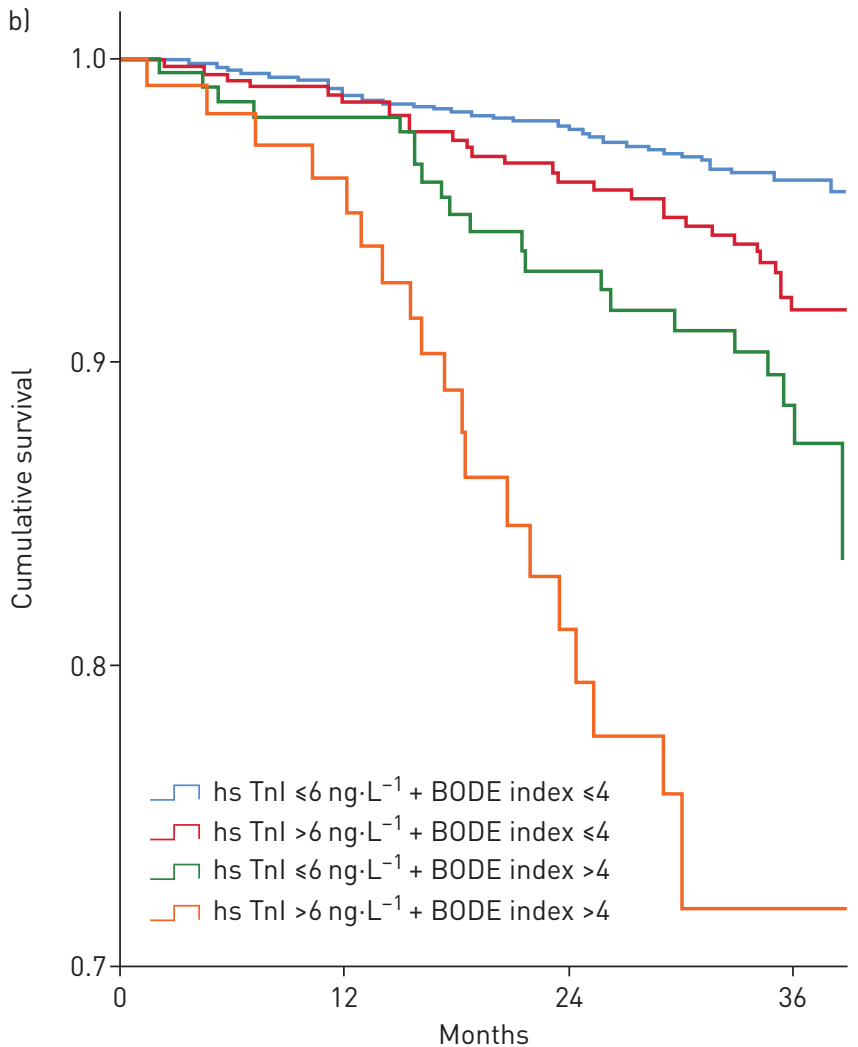

FIGURE 2 Kaplan-Meier survival curves of patients with stable chronic obstructive pulmonary disease (COPD) according to categorised variables combining high-sensitivity troponin I (hs-Tnl) and established COPD mortality predictors. a) hs-Tnl and forced expiratory volume in $1 \mathrm{~s}$ (FEV l $_{\text {l and }}$ b) hs-Tnl and the multidimensional BODE index (body mass index, airway obstruction, dyspnoea and exercise capacity), using the cut-offs hsTnl $6 \mathrm{ng} \cdot \mathrm{L}^{-1}, \mathrm{FEV}_{1} 50 \%$ pred and BODE score 4 (log-rank test $\mathrm{p}<0.001$ for a) and b)). Numbers at risk after 3 years of follow-up were $768,272,618$ and 251, respectively, for the categories in a), and 1150,409, 192 and 90, respectively, for the categories in b).

and hypertension in our cohort. Ours is the first study to evaluate such a wide range of cardiovascular and respiratory markers to independently predict higher levels of hs-TnI in patients with COPD. The present data clearly show that higher levels of hs-TnI in patients with COPD are predicted by a variety of different parameters that together reflect the broad spectrum of the disease.

Our most important observation was the predictive value of hs-TnI for all-cause mortality in this broad population. This report is the first in patients with stable COPD to find a strong association between hs-TnI and all-cause mortality. Our analyses showed that this association is independent from a range of established cardiovascular risk factors and prevalent cardiovascular diseases, as well as from the most relevant predictors of mortality in COPD, e.g. airflow limitation, poor exercise capacity, increased dyspnoea and a history of severe exacerbations. Thus, patients with hs-TnI levels $>6 \mathrm{ng} \cdot \mathrm{L}^{-1}$ had a 1.6 -fold higher risk of death over 3 years of follow-up compared to patients with levels $<6 \mathrm{ng} \cdot \mathrm{L}^{-1}$, even after adjusting for a wide range of other risk factors. We thereby clearly showed that hs-TnI concentrations that are well below the cut-off used for the diagnosis of myocardial infarction add prognostic information in stable COPD. To date, most studies on troponin in COPD have focused on patients with acute COPD exacerbations rather than stable disease $[34,35]$. To the best of our knowledge there are only two studies on the association between cardiac troponins and longitudinal outcomes in patients with stable COPD, and even these are in selected populations. First, in a small pilot study in patients with COPD who did not have overt cardiovascular disease, NeuKamm et al. [18] found a very high prevalence of elevated hs-troponin T levels (i.e. 24\%) which were associated with mortality. Second, a subgroup analysis of an interventional trial that included $>16000$ patients with moderate airflow limitation (GOLD stage II) and either a history of cardiovascular disease or increased cardiovascular risk also addressed hs-TnI and longitudinal outcomes [19]. In that post hoc analysis, ADAMSON et al. selected 1599 patients with an even higher prevalence of cardiovascular disease (three-quarters had an established cardiovascular disease or diabetes with end-organ damage) and found hs-TnI to be associated with cardiovascular events and cardiovascular death [19]. Our results are broadly in line with these previous studies, but extend the knowledge on the area. We clearly show that hs-TnI adds complementary prognostic information across 
all COPD severity stages and irrespective of the cardiovascular risk profile beyond a range of existing mortality predictors in COPD, including the components of the BODE index. For example, it is well known that all patients with COPD and a high BODE index are at high risk of all-cause mortality [22]. However, our patients with high BODE index and hs-TnI $>6 \mathrm{ng} \cdot \mathrm{L}^{-1}$ had a further increased risk of all-cause mortality, i.e. 2.6-fold higher compared to those patients with high BODE index but hs-TnI $<6 \mathrm{ng} \cdot \mathrm{L}^{-1}$. Furthermore, our results show that a correction for a wide range of cardiovascular risk factors and prevalent cardiovascular diseases, including an adjustment for NT-proBNP levels, attenuated the association between hs-TnI and mortality to some extent. Despite this, hs-TnI remained a strong and independent predictor for all-cause mortality. Of note, hs-TnI was a better predictor of mortality than NT-proBNP in our cohort.

A range of different mechanisms may account for the association of hs-TnI with COPD assessments and all-cause mortality independently. Troponin is a well-established indicator of myocyte damage and myocardial necrosis [9-11]. Slight increases in cardiac troponins can have both ischaemic and non-ischaemic causes [10, 36, 37]. Non-ischaemic mechanisms include endothelial dysfunction, inflammatory processes and increased myocardial strain due to pressure overload [36, 37]. We hypothesise that in a subset of patients with COPD, due to shared risk factors, the mechanism associated with higher troponin levels and a consequent increased mortality risk could be the same as that in patients with cardiovascular disease and no COPD. However, our secondary results indicate that other COPD associated factors could play an independent role for higher hs-TnI levels and a resultant elevated mortality risk in COPD, such as right ventricular dysfunction, hypoxaemia or exacerbations. Possible mechanistic pathways, all common in COPD, include systemic inflammation, endothelial dysfunction, neurohumoral activation and thrombogenic status [38]. Indeed, we observed an independent association between hs-TnI and hypoxaemia, which may be an explanation for a subset of COPD patients in our cohort. An indirect link to the role of neurohumoral activation may be the fact that dyspnoea grade and hypercapnia, two important drivers for heightened sympathetic activation in COPD [39], were also independent predictors of hs-TnI in the present report. Of note, the association between dyspnoea grade and hs-TnI was independent of NT-proBNP, and is therefore likely to represent the COPD-specific dyspnoea rather than the dyspnoea potentially caused by co-existing heart failure. Moreover, patients with COPD are known to be at high risk for a cardiovascular event or death during an exacerbation and during the post-exacerbation period [2, 40-42]. Our results suggest that a history of severe exacerbations is associated with higher hs-TnI levels even during the stable period. In turn, we and others did not find hs-TnI levels to predict future COPD exacerbations [19]. Under-treatment of cardiovascular diseases, which is often observed in patients with COPD, is unlikely to drive our results, since the frequency of classic cardiovascular medication increased according to hs-TnI quartiles in parallel with the increasing prevalence of cardiovascular diseases. Finally, low circulating levels of hs-TnI may indicate a very early pathology in myocytes in patients with COPD and the associated risk of death seems not to be covered by existing markers.

Our results have clear clinical relevance for patients with stable COPD. Hs-TnI is an objective assessment, which is available in most standard laboratories. Our data indicate that the additive use of hs-TnI improves risk stratification across a cohort of patients with mild to very severe COPD irrespective of their cardiovascular risk profile. Therefore, in clinical practice hs-TnI concentrations in the upper normal range may help the early identification of high-risk patients. Our data suggest that both respiratory and cardiovascular factors may play a role in higher hs-TnI values. In such patients, respiratory management should be evaluated and intensified (if not already optimised) first, including managing the risk of exacerbations and the control of symptoms. Second, these patients should be referred for additional cardiac assessments to identify potentially overlooked coexisting cardiovascular disease. This should at least include basic assessments such as blood pressure, ECG and lipids. As such, hs-TnI may help to guide appropriate treatment in order to reduce overall mortality in COPD.

Our study has several strengths. We describe the largest COPD cohort with hs-TnI measurements to date including patients across all severity stages and without any preselection according to cardiovascular risk profile. In addition, we conducted a very detailed cardiovascular characterisation for the first time in that context, with most variables based on objective measures. However, our analyses also have limitations. First, we do not have information on the cause of death, and so we cannot distinguish cardiovascular deaths from all-cause mortality. However, our primary aim was to study whether hs-TnI adds prognostic information independent from established predictors in general, which was the case here. Our data clearly show that the consideration of hs-TnI alongside established COPD predictors, such as the BODE index, improves risk stratification. Second, our analyses are based on hs-TnI measures from a single visit. Therefore, we cannot exclude the possibility that there could be intra-individual between-day variability in hs-TnI, leading to misclassification in some patients when using cut-offs, especially at the low end of the 
normal range [43]. However, ADAMSON et al. [19] reported in their study in patients with COPD that hs-TnI concentrations were unchanged during 3 months of follow-up. In addition, our main finding, that there is a continuous increase in mortality risk with increasing hs-TnI values beginning at low levels within the normal range, is unaffected by a misclassification bias. Furthermore, the results of our mortality analyses based on the existing cut-off of $6 \mathrm{ng} \cdot \mathrm{L}^{-1}$ are consistent with the results using hs-TnI as a continuous variable, which rules out a relevant misclassification bias.

In conclusion, our analyses showed that hs-TnI is a strong predictor of mortality in patients with COPD irrespective of their cardiovascular risk profile, with concentrations for increased risk well below the cut-off used for the diagnosis of myocardial infarction. This increased risk is independent from established mortality predictors of COPD. Circulating hs-TnI concentrations might help to identify high-risk COPD patients early in the course of the disease to facilitate earlier referral for intensified observation and specific treatment in the future.

Acknowledgements: The authors thank all patients of COSYCONET for their kind cooperation and all study centres for their excellent work. We are grateful to the scientific advisory board of COSYCONET for continuing support and helpful recommendations. We thank David Young (Young Medical Communications and Consulting Ltd, Horsham, UK) for a critical review; this was funded by the German Federal Ministry of Education and Research (BMBF) Competence Network Asthma and COPD (ASCONET). We also thank Francisco Ojeda (University Heart Center Hamburg, Hamburg, Germany) for statistical support.

Support statement: This work was supported by the German Federal Ministry of Education and Research (BMBF) Competence Network Asthma and COPD (ASCONET) and performed in collaboration with the German Centre for Lung Research (DZL). The project is funded by the BMBF with grant number 01 GI 0881, and is supported by unrestricted grants from AstraZeneca GmbH, Bayer Schering Pharma AG, Boehringer Ingelheim Pharma GmbH \& Co. KG, Chiesi GmbH, GlaxoSmithKline, Grifols Deutschland GmbH, MSD Sharp \& Dohme GmbH, Mundipharma $\mathrm{GmbH}$, Novartis Deutschland GmbH, Pfizer Pharma GmbH, Takeda Pharma Vertrieb GmbH \& Co. KG for patient investigations and laboratory measurements. The present analysis is also supported by the German Center for Cardiovascular Research (DZHK) under grant number 81Z1710101. The funding body had no involvement in the design of the study or the collection, analysis or interpretation of the data. Funding information for this article has been deposited with the Crossref Funder Registry.

Conflict of interest: B. Waschki has nothing to disclose. P. Alter reports grants from German Federal Ministry of Education and Research (BMBF) Competence Network Asthma and COPD (ASCONET), AstraZeneca GmbH, GlaxoSmithKline, Grifols Deutschland GmbH, MSD Sharp and Dohme GmbH, Pfizer Pharma GmbH and Takeda Pharma Vertrieb GmbH \& Co. KG, grants and non-financial support from Bayer Schering Pharma AG and Chiesi $\mathrm{GmbH}$, grants, personal fees and non-financial support from Boehringer Ingelheim Pharma GmbH \& Co. KG and Novartis Deutschland $\mathrm{GmbH}$, grants and personal fees from Mundipharma $\mathrm{GmbH}$, outside the submitted work. T. Zeller has nothing to disclose. C. Magnussen has nothing to disclose. J.T. Neumann reports personal fees from Abbott Diagnostics and Siemens, outside the submitted work. R. Twerenbold reports grants from Swiss National Science Foundation (grant number P300PB_167803), Swiss Heart Foundation, Swiss Society of Cardiology and Cardiovascular Research Foundation Basel, personal fees from Abbott Diagnostics, Amgen, Roche Diagnostics, Siemens, Singulex and Brahms, outside the submitted work. C. Sinning has nothing to disclose. C. Herr has nothing to disclose. K. Kahnert has nothing to disclose S. Fähndrich has nothing to disclose. S. Blankenberg reports personal fees from Abbott Diagnostics, Siemens, Thermo Fisher and Singulex outside the submitted work. K.F. Rabe has nothing to disclose. T. Welte reports personal fees for lectures and advisory board work from AstraZeneca, Boehringer, Berlin Chemie, Chiesi, GSK and Novartis, grants from AstraZeneca and Novartis, outside the submitted work. R.A. Jörres has nothing to disclose. C.F. Vogelmeier reports grants and personal fees from AstraZeneca, Boehringer Ingelheim, GlaxoSmithKline, Grifols and Novartis, personal fees from CSL Behring, Chiesi, Menarini, Mundipharma, Teva and Cipla, grants from Bayer-Schering, MSD and Pfizer, outside the submitted work. R. Bals reports grants and personal fees from AstraZeneca, Boehringer Ingelheim and Novartis, personal fees from GlaxoSmithKline, Grifols and CSL Behring, grants from German Federal Ministry of Education and Research (BMBF) Competence Network Asthma and COPD (ASCONET), Sander Stiftung, Schwiete Stiftung, Krebshilfe and Mukoviszidose eV, outside the submitted work. H. Watz reports personal fees from AstraZeneca, Boehringer Ingelheim, GlaxoSmithKline, BerlinChemie, Chiesi, Novartis and Roche, outside the submitted work.

\section{References}

1 Vogelmeier CF, Criner GJ, Martinez FJ, et al. Global Strategy for the Diagnosis, Management, and Prevention of Chronic Obstructive Lung Disease 2017 report. GOLD executive summary. Am J Respir Crit Care Med 2017; 195: 557-582.

2 Rabe KF, Watz H. Chronic obstructive pulmonary disease. Lancet 2017; 389: 1931-1940.

3 Rabe KF, Hurst JR, Suissa S. Cardiovascular disease and COPD: dangerous liaisons? Eur Respir Rev 2018; 27 : 180057.

4 Chen W, Thomas J, Sadatsafavi M, et al. Risk of cardiovascular comorbidity in patients with chronic obstructive pulmonary disease: a systematic review and meta-analysis. Lancet Respir Med 2015; 3: 631-639.

5 Anthonisen NR, Skeans MA, Wise RA, et al. The effects of a smoking cessation intervention on 14.5-year mortality: a randomized clinical trial. Ann Intern Med 2005; 142: 233-239.

6 Calverley PM, Anderson JA, Celli B, et al. Salmeterol and fluticasone propionate and survival in chronic obstructive pulmonary disease. N Engl J Med 2007; 356: 775-789.

7 Waschki B, Kirsten A, Holz O, et al. Physical activity is the strongest predictor of all-cause mortality in patients with COPD: a prospective cohort study. Chest 2011; 140: 331-342. 
8 Williams MC, Murchison JT, Edwards LD, et al. Coronary artery calcification is increased in patients with COPD and associated with increased morbidity and mortality. Thorax 2014; 69: 718-723.

9 Keller T, Zeller T, Peetz D, et al. Sensitive troponin I assay in early diagnosis of acute myocardial infarction. N Engl J Med 2009; 361: 868-877.

10 Thygesen K, Alpert JS, Jaffe AS, et al. Fourth universal definition of myocardial infarction (2018). Eur Heart $J$ 2019; 40: 237-269.

11 Westermann D, Neumann JT, Sörensen NA, et al. High-sensitivity assays for troponin in patients with cardiac disease. Nat Rev Cardiol 2017; 14: 472-483.

12 Neumann JT, Twerenbold R, Ojeda F, et al. Application of high-sensitivity troponin in suspected myocardial infarction. N Engl J Med 2019; 380: 2529-2540.

13 Apple FS, Murakami MM, Pearce LA, et al. Predictive value of cardiac troponin I and T for subsequent death in end-stage renal disease. Circulation 2002; 106: 2941-2945.

14 Eggers KM, Lagerqvist B, Venge P, et al. Persistent cardiac troponin I elevation in stabilized patients after an episode of acute coronary syndrome predicts long-term mortality. Circulation 2007; 116: 1907-1914.

15 Horwich TB, Patel J, MacLellan WR, et al. Cardiac troponin I is associated with impaired hemodynamics, progressive left ventricular dysfunction, and increased mortality rates in advanced heart failure. Circulation 2003; 108: 833-838.

16 Zeller T, Tunstall-Pedoe H, Saarela O, et al. High population prevalence of cardiac troponin I measured by a high-sensitivity assay and cardiovascular risk estimation: the MORGAM Biomarker Project Scottish Cohort. Eur Heart J 2014; 35: 271-281.

17 Blankenberg S, Salomaa V, Makarova N, et al. Troponin I and cardiovascular risk prediction in the general population: the BiomarCaRE consortium. Eur Heart J 2016; 37: 2428-2437.

18 Neukamm A, Einvik G, Didrik Høiseth A, et al. The prognostic value of measurement of high-sensitive cardiac troponin $\mathrm{T}$ for mortality in a cohort of stable chronic obstructive pulmonary disease patients. BMC Pulm Med 2016; 16: 164 .

19 Adamson PD, Anderson JA, Brook RD, et al. Cardiac troponin I and cardiovascular risk in patients with chronic obstructive pulmonary disease. J Am Coll Cardiol 2018; 72: 1126-1137.

20 Karch A, Vogelmeier C, Welte T, et al. The German COPD cohort COSYCONET: aims, methods and descriptive analysis of the study population at baseline. Respir Med 2016; 114: 27-37.

21 Zeller T, Ojeda F, Brunner FJ, et al. High-sensitivity cardiac troponin I in the general population - defining reference populations for the determination of the 99th percentile in the Gutenberg Health Study. Clin Chem Lab Med 2015; 53: 699-706.

22 Celli BR, Cote CG, Marin JM, et al. The body-mass index, airflow obstruction, dyspnea, and exercise capacity index in chronic obstructive pulmonary disease. N Engl J Med 2004; 350: 1005-1012.

23 Lucke T, Herrera R, Wacker M, et al. Systematic analysis of self-reported comorbidities in large cohort studies - a novel stepwise approach by evaluation of medication. PLoS One 2016; 11: e0163408.

24 Piepoli MF, Hoes AW, Agewall S, et al. 2016 European Guidelines on cardiovascular disease prevention in clinical practice: the Sixth Joint Task Force of the European Society of Cardiology and Other Societies on Cardiovascular Disease Prevention in Clinical Practice (constituted by representatives of 10 societies and by invited experts). Developed with the special contribution of the European Association for Cardiovascular Prevention \& Rehabilitation (EACPR). Eur Heart J 2016; 37: 2315-2381.

25 Levey AS, Stevens LA, Schmid CH, et al. A new equation to estimate glomerular filtration rate. Ann Intern Med 2009; 150: 604-612.

26 Houben-Wilke S, Jörres RA, Bals R, et al. Peripheral artery disease and its clinical relevance in patients with chronic obstructive pulmonary disease in the COPD and Systemic Consequences-Comorbidities Network Study. Am J Respir Crit Care Med 2017; 195: 189-197.

27 Fowkes FG, Murray GD, Butcher I, et al. Ankle brachial index combined with Framingham Risk Score to predict cardiovascular events and mortality: a meta-analysis. JAMA 2008; 300: 197-208.

28 Ponikowski P, Voors AA, Anker SD, et al. 2016 ESC Guidelines for the diagnosis and treatment of acute and chronic heart failure: the task force for the diagnosis and treatment of acute and chronic heart failure of the European Society of Cardiology (ESC). Developed with the special contribution of the Heart Failure Association (HFA) of the ESC. Eur Heart J 2016; 37: 2129-2200.

29 Alter P, Jörres RA, Watz $\mathrm{H}$, et al. Left ventricular volume and wall stress are linked to lung function impairment in COPD. Int J Cardiol 2018; 261: 172-178.

30 Lang RM, Badano LP, Mor-Avi V, et al. Recommendations for cardiac chamber quantification by echocardiography in adults: an update from the American Society of Echocardiography and the European Association of Cardiovascular Imaging. J Am Soc Echocardiogr 2015; 28: 1-39.

31 Celli BR, Locantore N, Yates J, et al. Inflammatory biomarkers improve clinical prediction of mortality in chronic obstructive pulmonary disease. Am J Respir Crit Care Med 2012; 185: 1065-1072.

32 Neukamm AM, Høiseth AD, Hagve TA, et al. High-sensitivity cardiac troponin T levels are increased in stable COPD. Heart 2013; 99: 382-387.

33 Hattori $\mathrm{K}$, Ishii $\mathrm{T}$, Motegi $\mathrm{T}$, et al. Relationship between serum cardiac troponin $\mathrm{T}$ level and cardiopulmonary function in stable chronic obstructive pulmonary disease. Int J Chron Obstruct Pulmon Dis 2015; 10: 309-320.

34 Brekke $\mathrm{PH}$, Omland $\mathrm{T}$, Holmedal SH, et al. Troponin $\mathrm{T}$ elevation and long-term mortality after chronic obstructive pulmonary disease exacerbation. Eur Respir J 2008; 31: 563-570.

35 Høiseth $\mathrm{AD}$, Neukamm A, Karlsson BD, et al. Elevated high-sensitivity cardiac troponin $\mathrm{T}$ is associated with increased mortality after acute exacerbation of chronic obstructive pulmonary disease. Thorax 2011; 66: 775-781.

36 Omland T, de Lemos JA, Sabatine MS, et al. A sensitive cardiac troponin T assay in stable coronary artery disease. N Engl J Med 2009; 361: 2538-2547.

37 Wallace TW, Abdullah SM, Drazner MH, et al. Prevalence and determinants of troponin T elevation in the general population. Circulation 2006; 113: 1958-1965.

38 Maclay JD, MacNee W. Cardiovascular disease in COPD: mechanisms. Chest 2013; 143: 798-807.

39 Andreas S, Anker SD, Scanlon PD, et al. Neurohumoral activation as a link to systemic manifestations of chronic lung disease. Chest 2005; 128: 3618-3624. 
40 MacDonald MI, Shafuddin E, King PT, et al. Cardiac dysfunction during exacerbations of chronic obstructive pulmonary disease. Lancet Respir Med 2016; 4: 138-148.

41 Suissa S, Dell'Aniello S, Ernst P. Long-term natural history of chronic obstructive pulmonary disease: severe exacerbations and mortality. Thorax 2012; 67: 957-963.

42 Donaldson GC, Hurst JR, Smith CJ, et al. Increased risk of myocardial infarction and stroke following exacerbation of COPD. Chest 2010; 137: 1091-1097.

43 Kavsak PA, Millar E, McLay G, et al. Between-day versus within-day imprecision using the Abbott high-sensitivity cardiac troponin I assay at concentrations around $5 \mathrm{ng} / \mathrm{l}$. Clin Chim Acta 2019; 489: 58-60. 